\title{
Mouvements verticaux en milieu marin faiblement stratifié
}

\author{
par J.-C. Gascard \\ Laboratoire d'Océanographie Physique du Muséum National \\ d'Histoire Naturelle
}

Nous avons pu observer une différence notable de la dynamique marine des eaux méditerranéennes, au large du golfe du Lion, entre l'été et l'hiver. Cette différence tient essentiellement au fait qu'en été, on se trouve en présence d'une stratification en densité très marquée par la présence de la thermocline, alors qu'en hiver cette stratification tend à disparaître sous l'effet du refroidissement et de l'évaporation.

En été, J. Gonella [1] a observé de fortes oscillations d'inertie engendrées par le vent dans la couche mélangée de surface, au-dessus de la thermocline. La thermocline semble agir comme un écran qui retiendrait la majeure partie de l'énergie cinétique, empruntée au vent, dans la couche de surface.

En hiver, par contre, en présence d'une faible stratification, cette énergie cinétique apparaît plus largement distribuée à travers la masse fluide. Les caractéristiques spectrales des mouvements montrent que l'énergie est alors également répartie entre l'énergie potentielle et l'énergie cinétique, dans une bande de fréquence qui va de la fréquence de stabilité (ou fréquence de Brunt-Väisälä) à la fréquence de Coriolis. Ce résultat indique que, dans cette bande spectrale, les mouvements sont alors régis par une dynamique d'ondes internes (ondes inertio-gravitationnelles).

\section{Ondes internes de stabilité}

Lors des missions Médoc en 1970, 1972, 1973, nous avons pu observer [2], en milieu marin faiblement stratifié, la présence quasi-permanente, en profondeur, d'ondes internes de grande amplitude et de longue période dans cette région de la Méditerranée située à la convergence des vents de Mistral et de Tramontane.
Cette observation tient essentiellement au fait que, depuis 1970, nous sommes capables de mesurer la composante verticale du courant grâce à la réalisation, par les chercheurs de la Woods Hole Oceanographic Institution (USA) [3], de courantomètres spéciaux, basés sur le principe du flotteur de Swallow. C'est un flotteur qui peut se maintenir, de façon stable, à une immersion où il se trouve en équilibre de densité avec le milieu ambiant, grâce au fait qu'il est constitué par une enveloppe dont le coefficient de compressibilité est plus faible que celui de l'eau de mer. Sur cette enveloppe, sont fixées des pales inclinées de $45^{\circ}$ par rapport à l'horizontale, de façon à ce que l'ensemble enveloppe pales constitue une hélice à axe vertical. Cette hélice, sans pivot, est extrêmement sensible et capable de détecter des vitesses verticales de l'ordre d'une fraction de $\mathrm{mm} / \mathrm{s}$. Cet ensemble, muni d'un émetteur acoustique, peut être localisé dans l'espace au moyen d'une base d'hydrophones.

Nous présentons, sur la figure $1 \mathrm{a}$, un enregistrement effectué en 1972 par un tel instrument, dont la trajectoire horizontale est représentée figure 3 . Les « rotations》 représentent le nombre de tours de l'instrument sur lui-même, à la fois dans le sens positif et négatif, correspondant respectivement à des remontées ou des plongées d'eau. Le pas de l'hélice est de l'ordre de 1, c'est-à-dire qu'à une rotation de l'instrument correspond un déplacement vertical de $1 \mathrm{~m}$ d'eau.

Cet enregistrement nous montre des mouvements d'eau à caractère oscillatoire. L'analyse de ces résultats (fig. $1 \mathrm{~b}$, $c$ et d) indique une forte densité d'énergie cinétique verticale pour des oscillations de période voisine de $4 \mathrm{~h} 35 \mathrm{mn}$. De plus, la mesure de la température au niveau du flotteur nous permet, avec la détermination de la vitesse verticale du fluide, de calculer un flux thermique vertical. Ce flux apparaît dans le calcul du coefficient de cross-correlation température-vitesse pour $t=0$. Il est très faible, voire nul, 

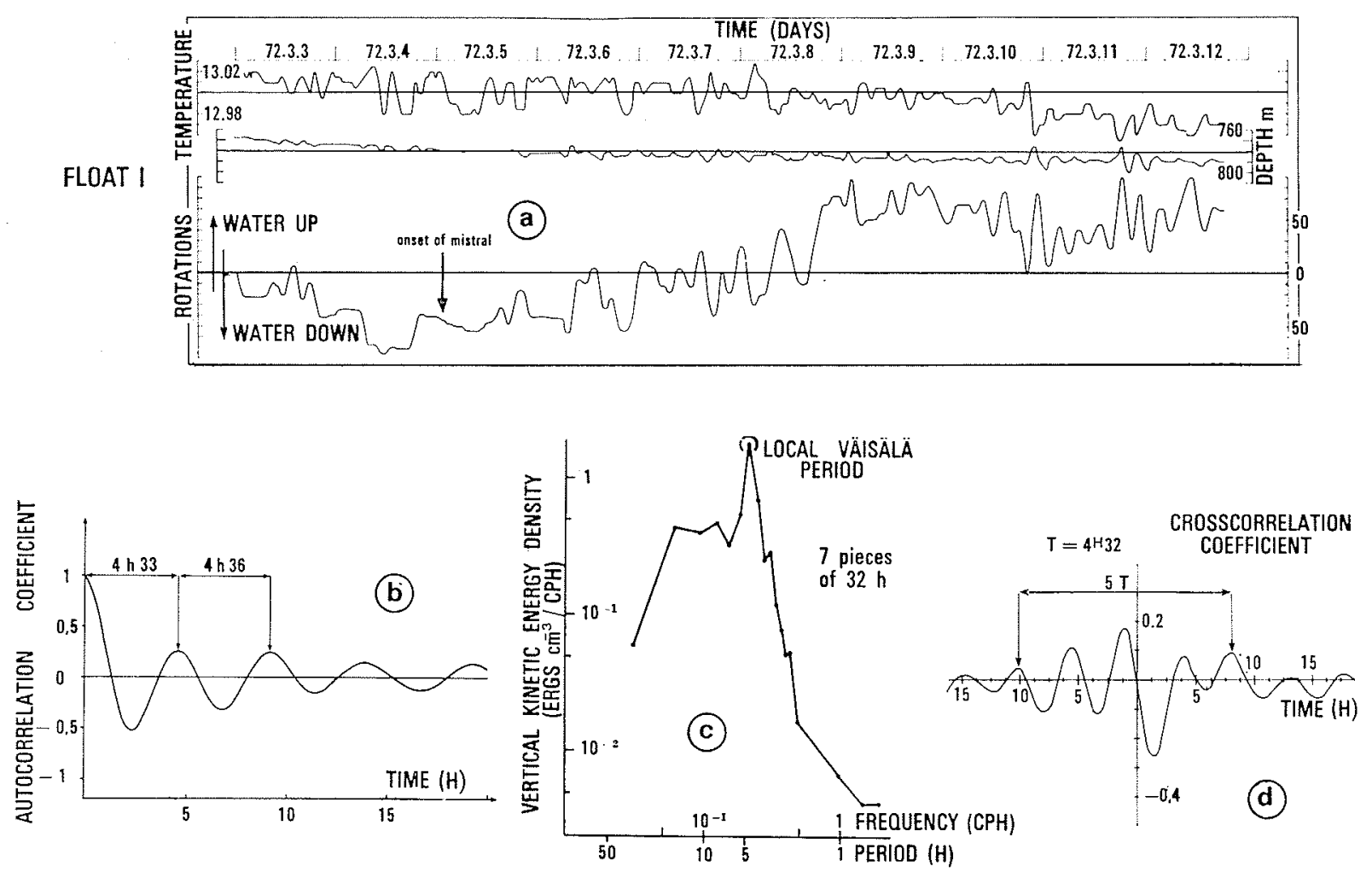

1/ a) Enregistrement interne de la température, pression et rotations du flotreur $n^{\circ} 1$ (Médoc 72); b, c, d) Traitement statistique des données enregistrées.
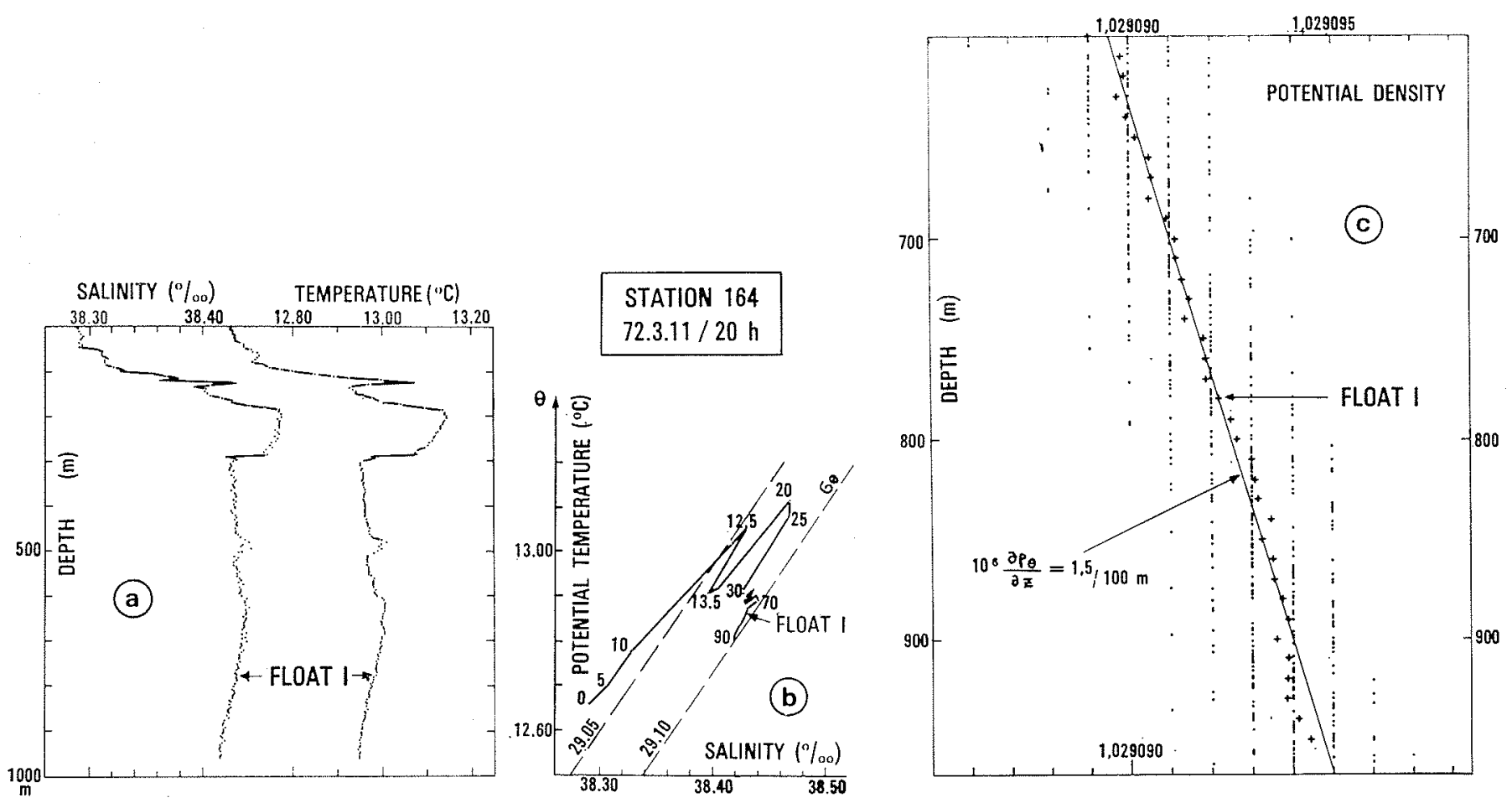

2/ Station hydrologique effectuée à la verticale du flotteur $n^{\circ} 1$ (Médoc 72). 


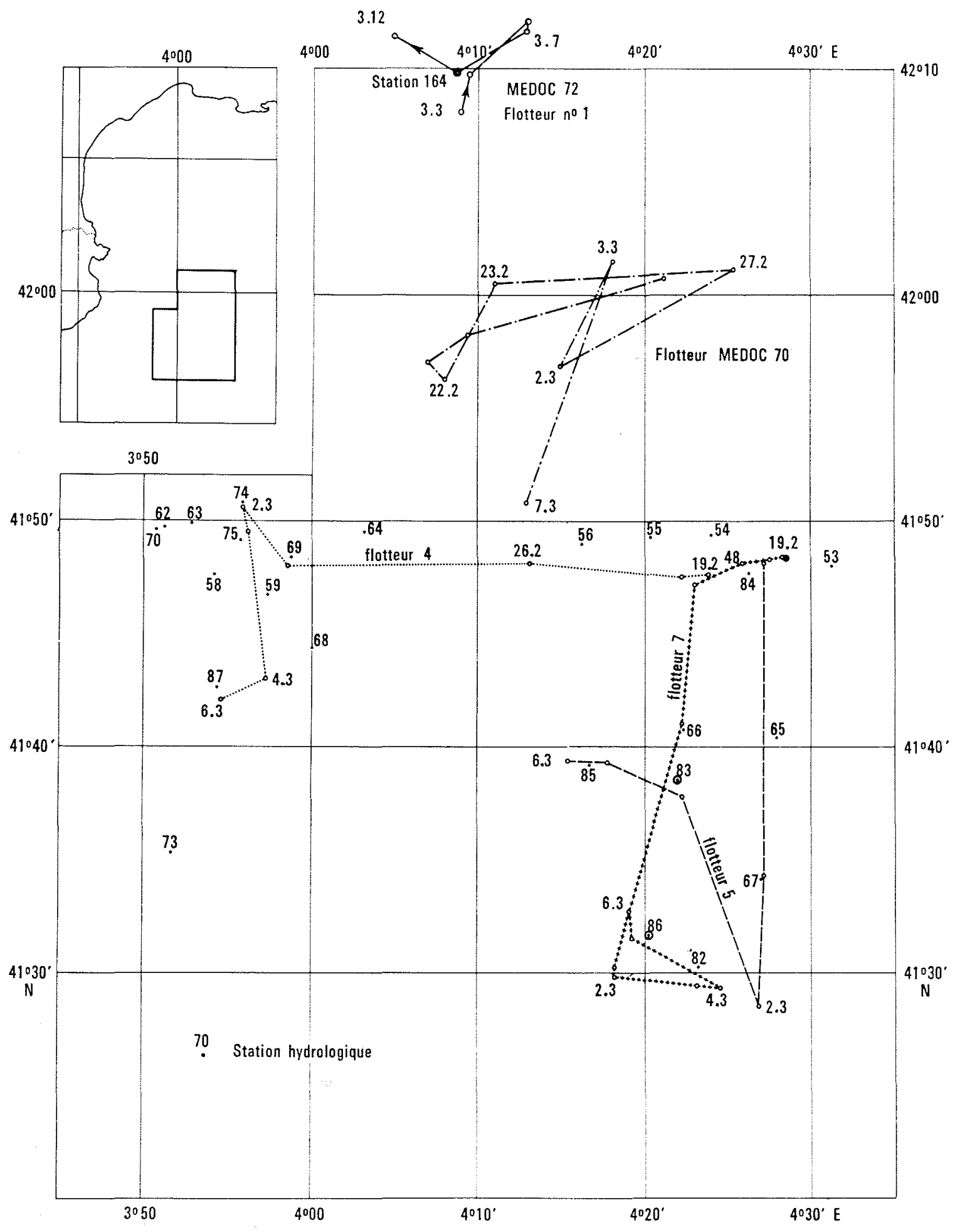

3/ Trajectoire des flotteurs 4,5 et 7 vers $800 \mathrm{~m}$ de profondeur, du 20 février au 6 mars 1973 (Médoc 73). 
à la précision de nos mesures. D'autre part, on retrouve une forte corrélation entre température et vitesse verticale à une période très proche de $4 \mathrm{~h} 35 \mathrm{mn}$. Cela signifie que ces oscillations sont caractéristiques d'une onde interne.

La mesure simultanée de la structure hydrologique du milieu au moyen de la bathysonde (fig. 2 a) nous a permis de calculer le gradient moyen de densité potentielle $\partial \rho_{G} / \partial z$ (fig. $2 \mathrm{c}$ ), dans la région où les enregistrements précédents de vitesse verticale et de température ont été obtenus. A partir de ce gradient, il nous est possible de déterminer la période de stabilité, autrement dit la période de BruntVäisälä

$$
\begin{aligned}
T_{\theta} & =\frac{2 \pi}{\sqrt{g / \rho_{\theta} \cdot \partial \rho_{\theta} / \partial z}}=4 \mathrm{~h} 37 \\
\operatorname{avec}\left|\frac{\partial \rho_{\theta}}{\partial z}\right| & =1,5 \cdot 10^{-8} \mathrm{~g} / \mathrm{cm}^{3} / \mathrm{m}
\end{aligned}
$$

Cette période, très proche de celle obtenue précédemment, nous permet de dire que l'onde interne mesurée est une onde de stabilité.

\section{Comparaison des composantes verticale et horizontale du courant}

\author{
(énergie cinétique, énergie potentielle)
}

Pendant l'hiver de 1973, nous avons eu l'occasion de comparer les résultats des mesures de courant obtenues à partir de trois flotteurs en dérive vers $800 \mathrm{~m}$ de profondeur et des mesures de courant à trois niveaux, au point fixe, obtenues à partir de courantomètres Aanderaa. Nous présentons, sur la figure 4, les hodographes intégrés de ces trois Aanderaa, hodographes qui montrent, entre 400 et $1200 \mathrm{~m}$, une remarquable cohérence du courant, dont la vitesse moyenne se situe entre 10 et $15 \mathrm{~cm} / \mathrm{s}$. Ce courant, portant vers le sud-ouest du 22 au 26 février, tourne de près de $90^{\circ}$ sur la droite à partir du 26 février. Nous présentons, sur la figure 3 et à la même échelle que la figure 4 , les trajectoires horizontales des trois flotteurs situés à une profondeur voisine de $800 \mathrm{~m}$. Il faut noter que les trois flotteurs 4, 5, 7 indiquent des orientations du courant moyen semblables à celles des Aanderaa, avec cependant des vitesses moyennes un peu plus faibles. Il est remarquable de noter que cette similitude se conserve sur des distances de l'ordre de 20 milles nautiques et des périodes de l'ordre d'une dizaine de jours (durée moyenne de nos enregistrements).

Si l'on observe un changement dans les hodographes le 26 février, rien de tel n'apparaît à la même date sur les trajectoires. Cette différence est probablement due à un phénomène d'advection. Par contre, le 2 mars, on remarque simultanément un changement radical des trois trajectoires et rien de semblable n'apparaît sur les hodographes. Cette comparaison est intéressante dans la mesure où elle permet de définir l'échelle, dans l'espace et dans le temps, de ces mouvements.
Pour ce qui est de la composante verticale du mouvement, nous présentons, figure 5, l'enregistrement obtenu à partir de l'un des trois flotteurs, où nous observons un maximum de $5 \mathrm{~cm} / \mathrm{s}$ le 28 février. Il nous est possible de comparer la répartition spectrale de la densité d'énergie cinétique entre la composante verticale et la composante horizonale du mouvement (fig. 6). Le niveau d'énergie cinétique vertical est supérieur à celui mesuré en 1972 et la fréquence de coupure (fréquence de Brunt-Väisälä) est inférieure, indiquant une stratification moins marquée. Ce résultat est confirmé par le profil hydrologique mesuré par la bathysonde (fig. 7).

Il est par ailleurs instructif de calculer l'énergie potentielle à partir de la composante verticale du mouvement, et de la comparer à l'énergie cinétique calculée à partir de la composante horizontale. En présence d'un gradient de densité potentielle $\partial \rho_{\theta} / \partial z$, les déplacements verticaux $\xi$ des particules fluides se traduisent par des variations d'énergie potentielle. L'énergie potentielle par unité de volume, pour un déplacement $\xi$, est le produit de la force de flottabilité moyenne 1/2 $g \Delta p_{\theta}$ par le déplacement $\xi$.

$$
1 / 2 g \Delta \rho_{\theta} \xi=1 / 2 \rho_{\theta} N^{2} \xi^{2}
$$

avec $N^{2}=-\left(g / \rho_{\theta}\right) .\left(\partial \rho_{\theta} / \partial z\right) . \quad N$ fréquence locale de Brunt-Väisälä

Soit $\left[\xi^{2}\right]$ le spectre en fréquence du déplacement vertical de l'eau. Donc, $E_{j}=1 / 2 \rho_{\theta} N^{2}\left[\xi^{2}\right]$ représente le spectre en fréquence de l'énergie potentielle.

Comme le spectre en fréquence de l'énergie cinétique de la composante verticale du mouvement est donné par:

$$
E_{c}^{\prime}=1 / 2 \rho_{\theta} \omega^{2}\left[\xi^{2}\right]
$$

on calcule directement le spectre d'énergie potentielle à partir du spectre d'énergie cinétique par:

$$
E_{p}=E_{c} \cdot\left(N^{2} / \omega^{2}\right)
$$

Les mesures indiquent que le spectre d'énergie potentielle a approximativement la même forme et la même amplitude que le spectre d'énergie cinétique horizontale. Il est significatif de rapprocher ce résultat du fait que:

$1^{\circ}$ on observe, dans le spectre de densité d'énergie cinétique de la composante horizontale du mouvement, une décroissance de l'énergie fonction du carré de la fréquence;

$2^{\circ}$ on observe, dans le spectre de densité d'énergie cinétique $E_{c}$ de la composante verticale, une répartition presque constante de la densité d'énergie à partir de la fréquence d'inertie; comme $N^{2}$ est aussi constante, $E_{p}$ est également une fonction en $f^{-2}$.

D'où on observe une distribution de l'énergie potentielle semblable à la distribution de l'énergie cinétique horizontale dans cette bande de fréquences (fig, 6).

\section{Remarque}

Si la fréquence de Brunt-Väisälä apparaît bien comme une fréquence de coupure dans les spectres d'énergie de la 

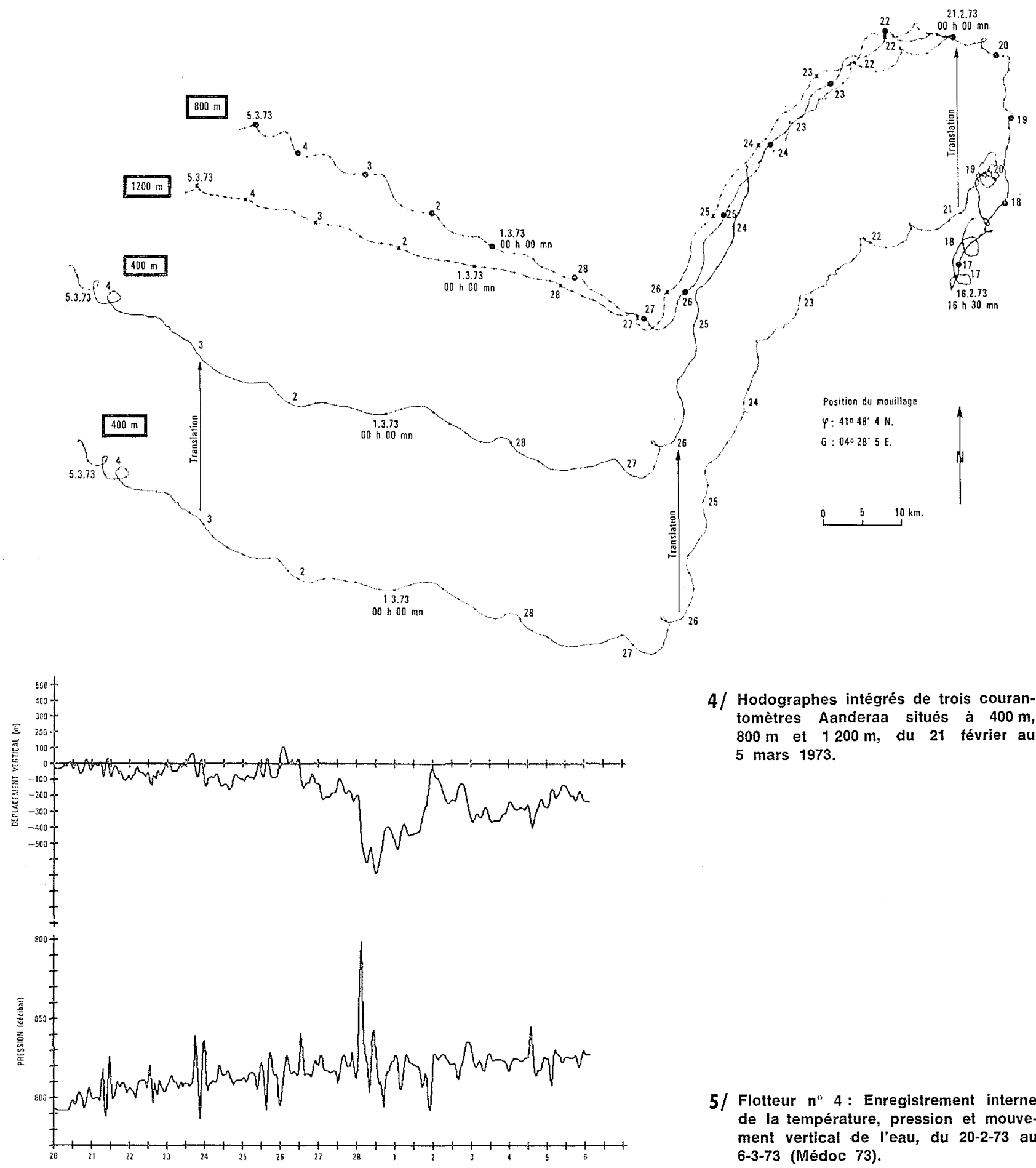

4/ Hodographes intégrés de trois courantomètres Aanderaa situés à $400 \mathrm{~m}$, $800 \mathrm{~m}$ ê $1200 \mathrm{~m}$, du 21 février au 5 mars 1973.

5/ Flotteur $n^{\circ} 4$ : Enregistrement interne de la température, pression et mouvement vertical de l'eau, du 20-2-73 au 6-3-73 (Médoc 73).

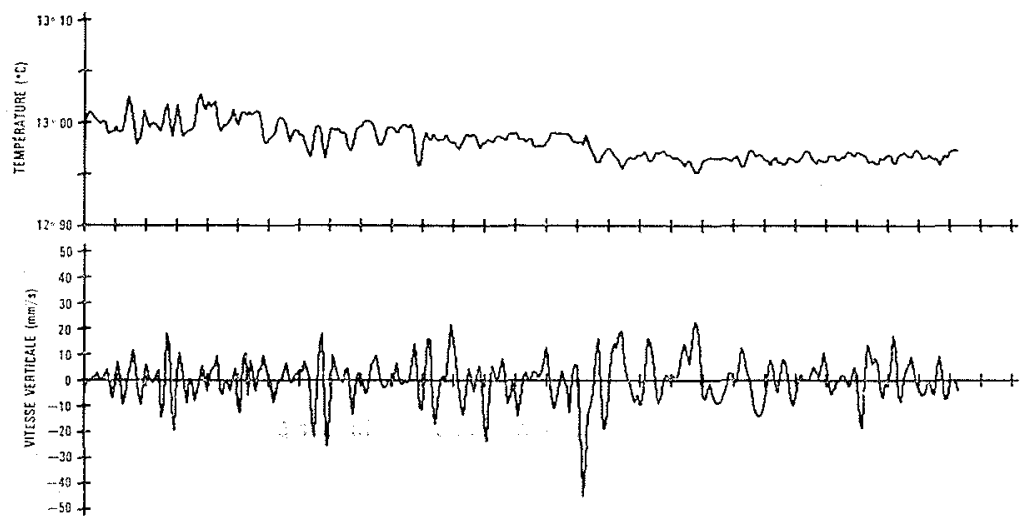




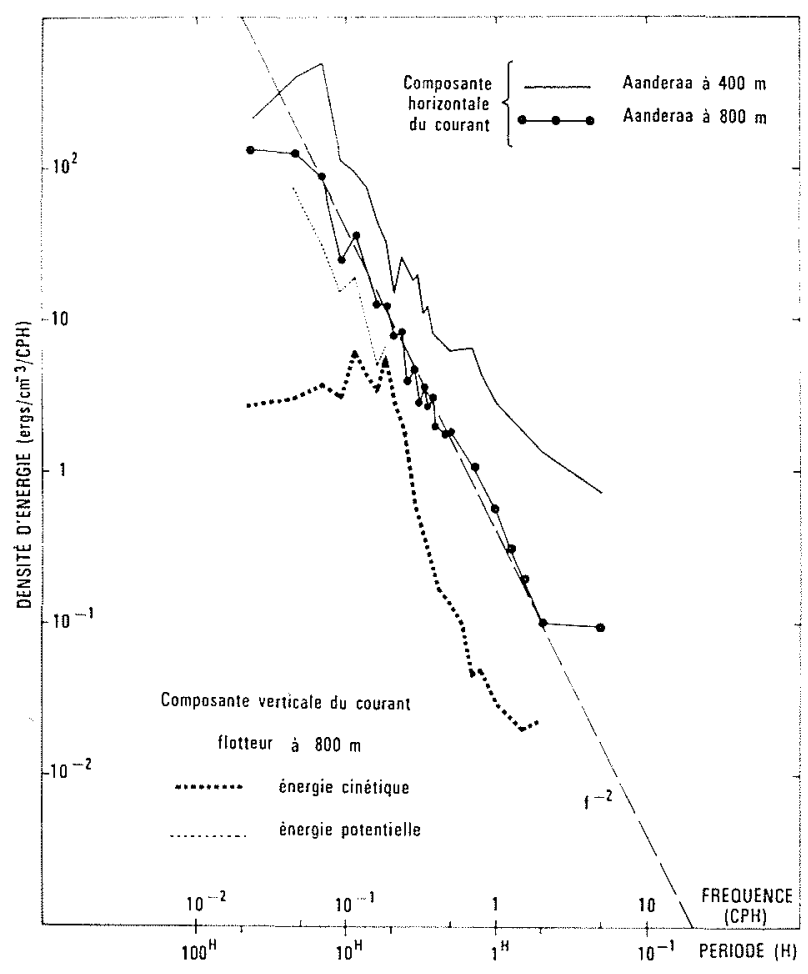
6/ Comparaison spectrale des densités d'énergie cinétique calculées d'après la mesure de la composante horizontale du courant à $400 \mathrm{~m}$ et $800 \mathrm{~m}$ et des densités d'énergie cinétique et poten- tielle calculées d'après la mesure de la composante verticale du courant à $800 \mathrm{~m}$ (Médoc 73).
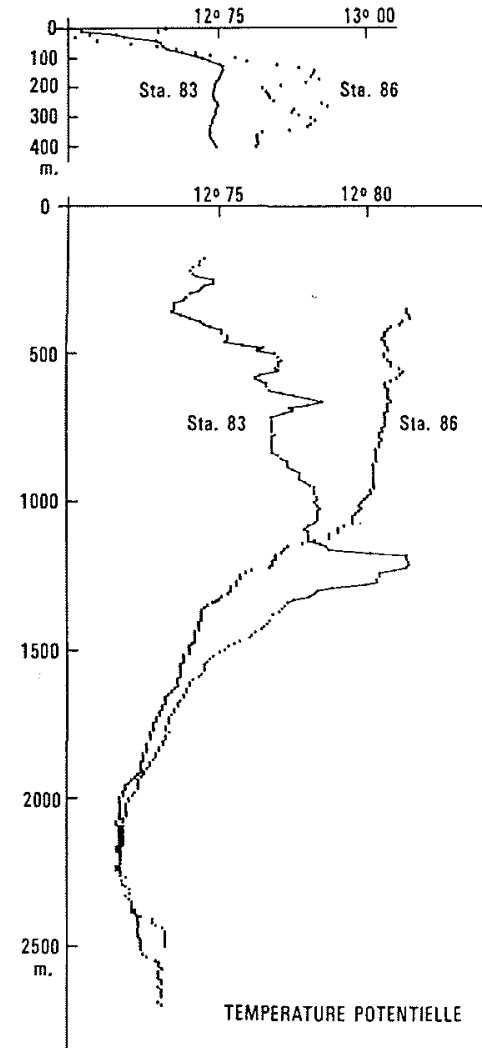
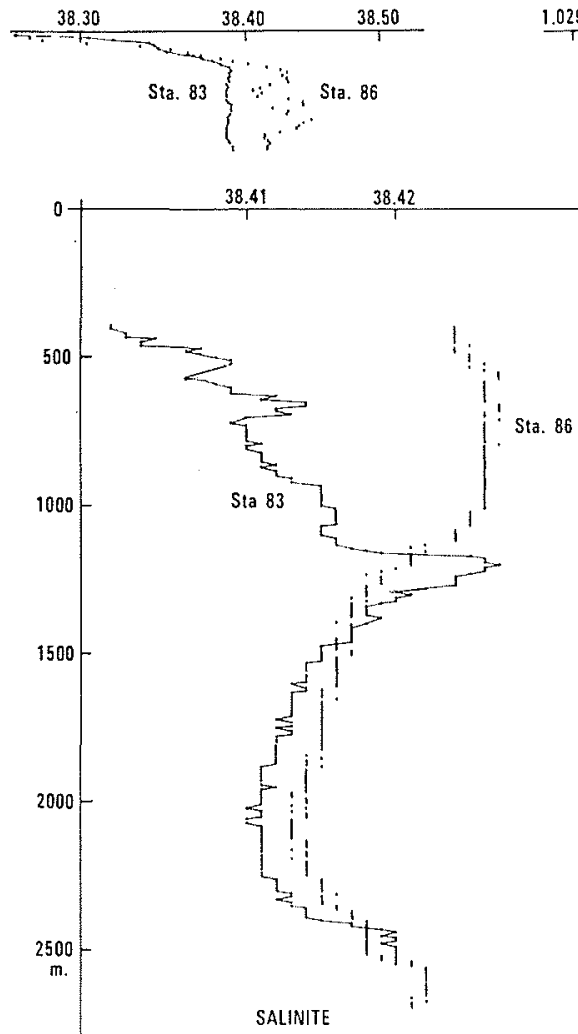
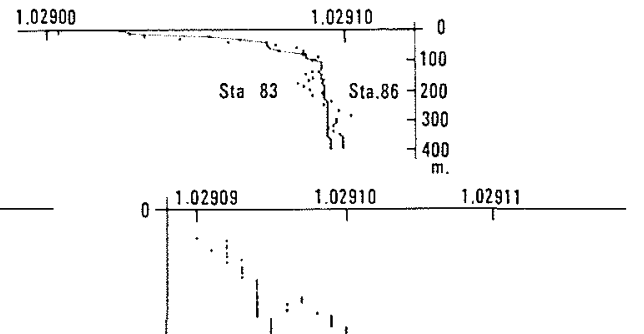

500

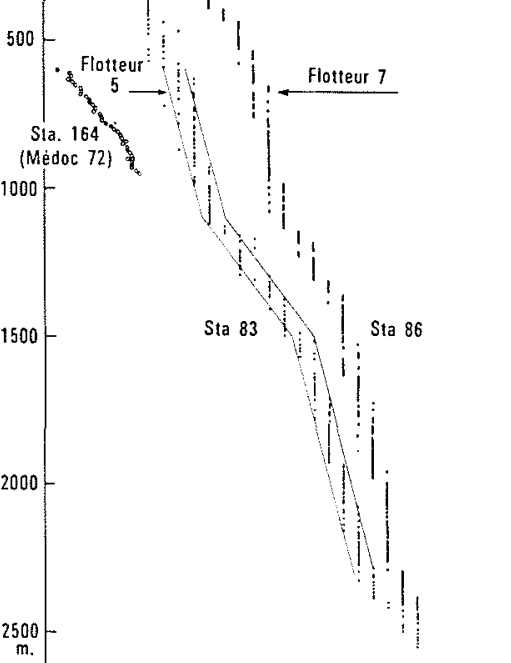

DENSITE POTENTIELLE
Station 83 , le 5.3 .1973 à 4 h 40 Station 86 , le 5.3 .1973 à $22 \mathrm{~h} 00$ Station 164, le 11.3.1972 à 20 h 00 $\varphi=41^{\circ} 38^{\prime} 5 \mathrm{~N}$. $c=41^{\circ} 31^{\prime} 6 \mathrm{~N}$. $c=42^{\circ} 09^{\prime} 8 \mathrm{~N}$
$G=4^{\circ}{ }^{\prime} 2^{\prime} \quad E$ à la verticale du Flotleur 5 $\mathrm{G}=4^{\circ} 20^{\prime} 2 \mathrm{E}$ à la verticale du Flotteur 7 $\mathrm{G}=4^{\circ} 08^{\prime} 7 \mathrm{E}$ à la verticale du Flotteur 5

7/ Comparaison des structures hydrologiques observées en 1973 et en 1972. 
composante verticale, nous ne pouvons rien conclure en ce sens pour la fréquence d'inertie. Ceci est dû à la duréc trop faible de nos enregistrements, qui ne nous permet pas de pousser de façon significative l'analyse statistique vers les longues périodes.

\section{Conclusion}

Les mouvements observés, représentatifs d'un ensemble d'ondes internes de nature inertio-gravitationnelles, sont donc régis par la dynamique de ces ondes.

Ce résultat est important quand on sait que, dans cette région de la Méditerranée, des mouvements convectifs très importants existent pendant la période hivernale, mouvements par lesquels se forment les eaux profondes méditerranéennes [4]. Nous avons eu l'occasion, en 1970, d'observer de tels phénomènes qui se manifestent par des courants verticaux pouvant atteindre $10 \mathrm{~cm} / \mathrm{s}$ [5]. Comment viennentils se superposer aux ondes internes que nous observons de façon presque systématique en hiver ? Existe-t-il une relation de cause à effet? Cette convection naturelle serait-elle déclenchée par un effet de résonance de l'onde de stabilité excitée par l'énergie empruntée aux vents, par exemple? C'est un problème que nous devons élucider si nous voulons comprendre cette mécanique très particulière de la convection océanique.

\section{Discussion}

Président : M. le Professeur H. Lacombe

En ouvrant la séance, $M$. le Président indique que le premier exposé de l'après-midi, intitulé «Mouvements verticaux en milieu marin faiblement stratifié », devait être présenté par M. GASCARD; celui-ci est, en ce moment, en mission en Méditerranée où il poursuit des études sur le même sujet; c'est un collègue de son laboratoire, M. Gonel, la, qui va, à sa place, présenter son exposé.

M. Gascard, confirme M. Gonella, m'a remis un certain nombre de diapositives que je vais commenter en essayant de rapporter auss fidèlement que possible ses conceptions; la conclusion de son étude me paraît être que, dans les zones de formation d'eau profonde, outre des actions de refroidissement et de convection, il peut $y$ avoir des phénomènes mécaniques extrêmement importants, liés aux processus de résonance avec la fréquence de Brunt-Väisälä; il peut en résulter parfois une certaine instabilité.

\section{$*$}

M. le Président remercie M. Gonfrla de cet exposé, axé sur le phénomène des vitesses verticales, encore peu familier aux spécialistes de la mer. It ouvre ensuite la discussion :

Après un échange de vues avec $M$. le Professeur Woons, M. le Président conclut :

En présence d'une stratification établie, comme en été par exemple, on a l'impression, comme l'a dit M. GoNella, que l'énergie, qui provient du vent et qui passe dans l'eau, sert essentiellement à provoquer des courants horizontaux sur la fréquence d'inertie; au contraire en période de quasi-neutralité de la stratification, il semble que l'énergie du vent passe essentiellement dans les courants verticaux.

C'est là un problème important. Peut-être l'un d'entre vous pourrait nous donner un début d'explication de co phénomène?

En hiver, commente M. SAINT-GuILy, la faiblesse de la stratification fait que l'action mécanique du vent, et l'action thermique due à

\section{Bibliographie}

[1] Gonelra (J.). - A local study of inertial oscillations in the upper layers of the ocean (1971). Deep-Sea Res., 18, pp. 775788.

[2] GASCARD (J.-C.). - Vertical motions in a region of deep water formation (1973). Deep-Sea Res., 20, pp. 1011-1027.

[3] Webb (D. C.), Dorson (D. L.) and Voorhis (A. D.). - A new instrument for the measurements of vertical currents in the ocean. Conference on Electronic Engineering in Ocean Technology, University College of Swansea, 21-24 sept. 1970 (1970). I.E.R.E. Proc., 19, pp. 323-331.

[4] Lacombe (H.) et TChernia (P.). - Le problème de la formation des eaux marines profondes (1972). Ann. Inst. Océanogr. Paris, 48 (1), pp, 75-110.

[5] Voorhis (A. D.) and WEBB (D. C.). - Large vertical currents observed in a winter sinking region of the northwestern Mediterranean (1970). Cah. Océanogr., 22, pp. 571-580. 
Pour M. Gonella, les deux cas susvisés semblent avoir été observés :

a) Un pic d'énergie dans les vitesses verticales à une fréquence égale à celle de Brunt-Väisälä, calculée à partir du gradient vertical de densité potentielle, prouve l'existence d'ondes internes se propageant horizontalement à cette fréquence.

b) Au cours des périodes où ont été observées les stratifications les plus faibles, les oscillations verticales de grande amplitude semblent beaucoup plus liées à des phénomènes de non-linéarités.

Le phénomène est tri-dimensionnel, estime $M$. SaINT-Guily, e il $\mathrm{y}$ a des dimensions spatiales associées à chaque onde; celles-ci dépendent du rapport de la profondeur aux diverses longueurs d'ondes présentes; le spectre de ces dernières présente un pic au droit de la fréquence théorique de déphasage.

M. le Président clôt la discussion, en ces termes :

M. GASCARD a rendu compte d'un certain nombre d'observations faites en Méditerranée; nous ignorons encore beaucoup d'aspects de ce genre de phénomènes et les mécanismes susceptibles de les interpréter ne sont pas connus.

Il donne ensuite la parole à $M$. Suberville pour l'exposé de la communication qu'il a rédigée avec $M$. ChaberT-b'Hières. Un film en couleurs illustre parfaitement les expériences effectuées par les auteurs sur la plaque tournante de Grenoble. 